\title{
Direction Selectivity in the Goldfish Tectum Revisited
}

\author{
VADIM MAXIMOV, ELENA MAXIMOVA, AND PAUL MAXIMOV \\ Institute for Information Transmission Problems, Russian Academy of Sciences, \\ Moscow, Russia
}

\begin{abstract}
Responses of direction-selective (DS) ganglion cells (GCs) were recorded extracellularly from their axon terminals in the superficial layer of the tectum opticum (TO) of immobilized goldfish, Carassius auratus gibelio Bloch. Directional tuning curves were measured with contrast edges moving in 12 or more different directions across the receptive field (RF). All directional tuning curves had cardioid-like appearance, their acceptance angles amounted to somewhat more than $\mathbf{1 8 0}^{\circ}$. According to their preferred directions DS GCs proved to comprise three distinct groups, each group containing DS GCs of ON and OFF subtypes approximately in equal quantity. Thus, this gives six physiological types of DS GCs in total. The preferred direction of a DS GC does not depend to some extent on a value of contrast, speed, size, and form of the stimuli. Coincidence in number of preferred directions with number of semicircular canals implies that DS GCs projecting to tectum are involved in some multimodal sensory integration in postural, locomotor, and oculomotor control in the three-dimensional aquatic world. DS neurons of the TO itself respond independently of the sign of stimulus contrast, have enormous receptive fields, and seem likely to collect signals from the retinal DS units of both ON and OFF subtypes with the same preferred direction.
\end{abstract}

KEYWORDS: direction-selective neurons; ganglion cells; ON and OFF pathways; tectum opticum; goldfish

\section{INTRODUCTION}

Direction-selective visual neurons respond optimally to stimulus motion in one (preferred) direction, but do not respond to motion in the opposite direction. DS ganglion cells were first described in the retinas of rabbit ${ }^{1}$ and goldfish ${ }^{2,3} 40$ years ago. The rabbit's DS GCs have been extensively studied since then (see review in Vaney and colleagues ${ }^{4}$ ). There are two distinct types of DS GCs that differ in their specificity for stimulus contrast, size, and speed and in their central projections. The most numerous ON-OFF type DS GCs are excited by stimuli that are lighter or darker than the background and project to the superior colliculus, while the ON type DS GCs are excited only by stimuli that are lighter than the background and project to the acces-

Address for correspondence: Dr. Vadim V. Maximov, Institute for Information Transmission Problems, Russian Academy of Sciences, 127994 Moscow GSP-4, Russia. Voice: 7-095-9523303; fax: 7-095-209-0579

maximov@iitp.ru

Ann. N.Y. Acad. Sci. 1048: 198-205 (2005). (C) 2005 New York Academy of Sciences. doi: 10.1196/annals.1342.018 
sory optic system. The ON-OFF cells, in turn, comprise four physiological subtypes with different preferred directions aligned with the horizontal and vertical ocular axes, whereas the ON cells comprise three physiological subtypes according to their preferred directions.

Fish were investigated less thoroughly. There are only few papers in which DS GCs of the fish retina have been mentioned. Although all authors noted the dominance of caudo-rostral preference in their recordings, $, 2,3,6-10$ no clear classification of these cells either by type of response (ON, OFF, or ON-OFF) or by preferred directions was made until now.

In our previous works with fairly rare color-coding units ${ }^{11,12}$ and detectors of oriented lines, ${ }^{13}$ projecting to the fish TO, we also regularly recorded responses from the DS units. Even without having to state the problem of classification of DS GCs (and not having technical conditions for this at that time), we had to know their properties for positioning in tectal layers during experiments. As a result, the following rules of thumb about DS units were formed. Unlike rabbit's mixed ON-OFF type of DS units, two separate types, pure ON and pure OFF units, were equally encountered in the fish TO. Responses of these types were recorded only in the most superficial part of the retinorecipient layer of the tectum. Units that prefer caudo-rostral direction of stimulus movement were most numerous and concentrated in the upper half of this sublayer. Below, DS units selective rather to the opposite direction of movement were located, though one could not exclude the existence of some DS units whose preferred direction was closer to vertical position rather than to horizontal. Also, without special investigations it was impossible to exclude the existence of ON-OFF DS units typical for the rabbit.

In the present study, we have systematically investigated the responsive and directional properties of some hundreds of DS GCs in the goldfish retina. Our results indicate that goldfish has six physiologically distinct subtypes of DS GCs essentially different from those of rabbit. Some of the results reported here have appeared in abstract form. ${ }^{14}$

\section{METHODS}

Responses of DS GCs were recorded extracellularly from their axon terminals in the superficial layer of the TO of immobilized goldfish, subspecies Carassius auratus gibelio Bloch, 10-15 cm standard body length, placed in a transparent Plexiglas tank with aerated water perfused through its gills. Visual stimuli (edges, bars, and spots of different brightness moving with different speed in different directions) were presented at the computer-controlled CRT monitor to the right eye of the fish.

Low impedance $(200-500 \mathrm{kOhm})$ recording microelectrodes were made of micropipettes filled with a Wood's metal and tipped with a platinum cap of diameter 2$10 \mu \mathrm{m} .{ }^{15}$ When a unit was isolated and an approximate position of its receptive field (RF) was found, a polar response pattern (directional tuning curve) of the unit was measured with contrast edges moving in 12 or more different directions across the RF. Mean number of spikes, $N$, in the response (over several repeated runs in each direction) as a function of direction was approximated by a second-order harmonic function: 


$$
N(\varphi)=a_{0}+a_{1} \cdot \cos \left(\varphi-\varphi_{1}\right)+a_{2} \cdot \cos \left(2 \varphi-2 \varphi_{2}\right)
$$

The amplitudes of the zero $\left(a_{0}\right)$, first $\left(a_{1}\right)$, and second $\left(a_{2}\right)$ harmonics, and the phases of the first $\left(\varphi_{1}\right)$ and second $\left(\varphi_{2}\right)$ harmonics characterize the polar response patterns.

The rostro-caudal direction was taken as the reference one. Other directions of movement were measured as polar angles relative to the reference direction, considered positive for counterclockwise rotation of the vector.

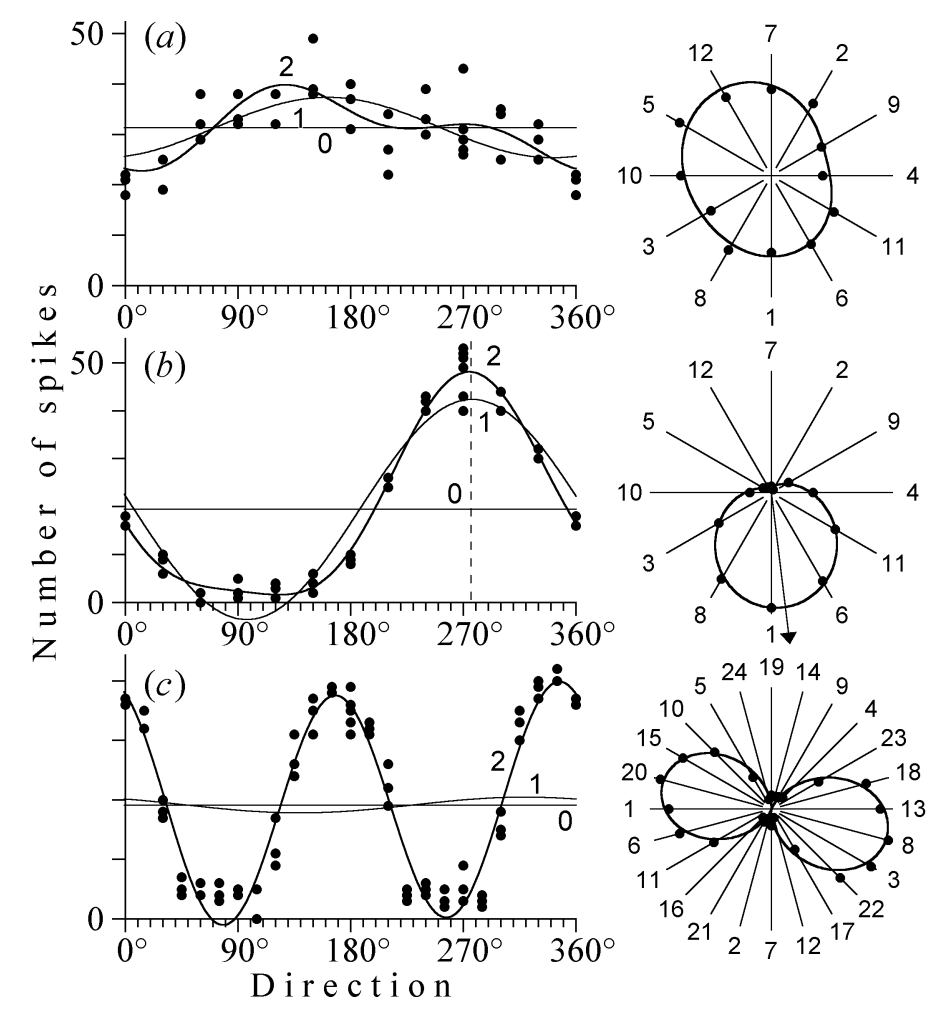

FIGURE 1. Examples of three principal types of polar response patterns. Diagrams were plotted in Cartesian (left) and polar (right) coordinates using the same experimental data. Dots mark number of spikes in the response either separately for each run in each direction as an ordinate value in the Cartesian plots, or a mean value over repeated runs as a radius in the polar plots. Solid lines are approximations by harmonic functions of zero (0), first (1), and second (2) order (only second-order approximations are shown in the polar plots). Numbers at the ends of radius-vectors in the polar plot indicate a sequence of presentations of different directions. Types of units and stimulation: (a) "dark spot detector," responses to movement of black stripes against a white background; $(b)$ direction-selective unit of the OFF type selective to dorso-ventral movement (preferred direction is shown by a dashed line in the Cartesian plot and by an arrow in the polar plot), responses to black edges moved in 12 directions against a white background; $(c)$ "detector of vertical line" (orientation-selective unit), responses to white stripes moved in 24 directions against a black background. 


\section{RESULTS AND DISCUSSION}

\section{Classification of Ganglion Cells by Their Polar Response Patterns}

It is customary to divide visual neurons by their polar response patterns into nonselective, direction-selective, and orientation-selective. ${ }^{1,7,16}$ Typical examples of polar response patterns measured for different GCs projecting to the fish TO are shown in FIGURE 1. Three types differ from each other by relative contribution of different harmonics. The amplitudes of the first and second harmonics reflect the strengths of the directional and orientational components, respectively, ${ }^{16}$ and, so, can be considered as classifying features. As one can see from the distribution shown in FIGURE 2, according to their polar patterns, GCs projecting to the tectum indeed constitute three clear clusters: (1) those with small relative amplitudes of both first and second harmonics - non-selective units; (2) those with pronounced relative amplitude of the first harmonic_-direction-selective units; (3) those with pronounced relative amplitude of the second harmonic_-orientation-selective units. DS units that according to this classification fall into the domain, where $a_{1}>\frac{1}{2} a_{0}$ and $a_{1}>a_{2}$, were subjects of the present study.

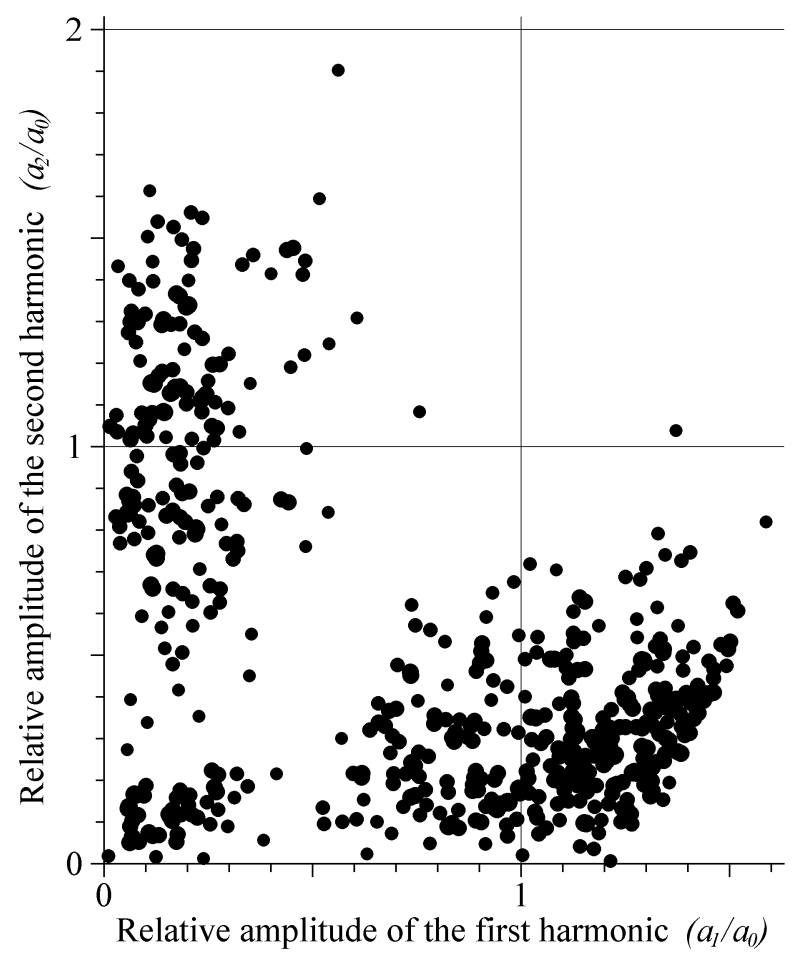

FIGURE 2. Scatter diagram of amplitude of the second harmonic versus amplitude of the first one for 522 polar patterns measured for GCs of various types. 


\section{Classification of DS GCs by Their Selectivity to Sign of Contrast}

As a rule even during the course of searching of units, the experimenter understands rather easily what a moving edge excites the recorded DS GC better: the light one against the dark background or quite the opposite. To get quantitative estimates when comparing responses to moving edges of opposite contrast the following experimental paradigm was used. A white or black bar that exceeded the RF in width were moved in preferred direction against a black or white background, respectively. Numbers of spikes in response to its leading and trailing edges were counted. The degree of selectivity to sign of contrast was estimated according to the ratio:

$$
\text { ratio }=\frac{N_{W / B}-N_{B / W}}{N_{W / B}+N_{B / W}},
$$

where $N_{W / B}$ and $N_{B / W}$ are numbers of spikes in response to white-black and blackwhite contrast edges, respectively. The bimodal distribution of the ratio shown at the histogram of FIGURE 3 indicates that there are two equally encountered principal types of DS GCs projecting to the goldfish TO: (1) ON type, which responds mainly at the introduction of a light stimulus into the RF, (2) OFF type, which responds mainly at the introduction of a dark stimuli.

In full agreement with this classification by moving contrast edges, different types of DS GCs respond also in a different way to small stationary spots turned on or off in the central region of their RF: the ON cells respond predominantly to the onset of illumination, whereas the OFF cells respond predominantly to its off-set.

So, in contrast to the rabbit with only one ON-OFF type of DS GCs projecting to the superior colliculus (tectum opticum), the goldfish turned out to have two equally encountered principal types of DS GCs projecting to its tectum.

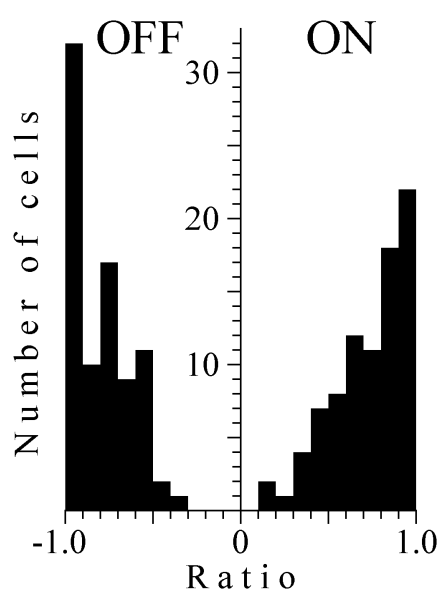

FIGURE 3. Distribution of the ratio describing the degree of selectivity to sign of contrast (see text) of moving edge for 167 selective recordings from DS $\mathrm{GC}$ endings. 


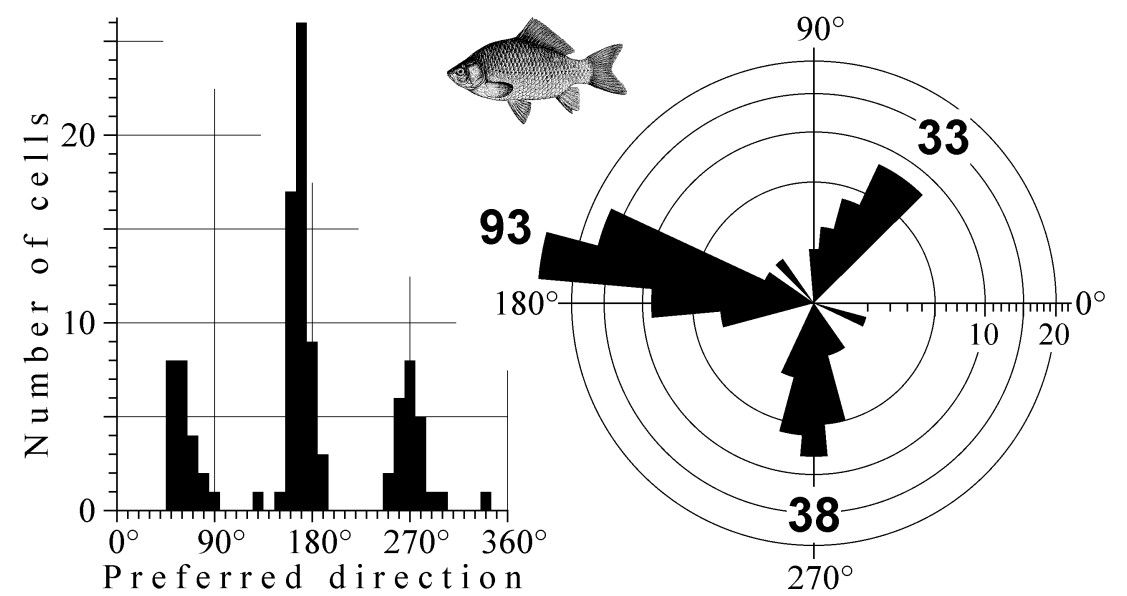

FIGURE 4. Distribution of preferred direction plotted in Cartesian (left) and polar (right) coordinates.

\section{Classification of DS GCs by Preferred Directions}

The preferred direction was defined as a phase of the first harmonic $\left(\varphi_{1}\right)$ of the Fourier transform that is equivalent to the orientation of the vector sum of the responses to all directions of motion (FIG. $1 \mathrm{~b}$ ). It was shown that the preferred direction of a DS GC does not depend to some extent on a value of contrast, speed, size, and form of the stimuli. In order to determine if there was any subdivision of DS GCs into physiological types by preferred direction, more than 150 well-isolated recordings from different DS GCs were selected from our experimental database of polar response patterns. Histograms of the preferred directions of these units are shown in FIGURE 4 both in Cartesian and polar coordinates. Although the sample comprised the units with different locations of their RF in the visual space, the histograms revealed obvious grouping. According to their preferred directions DS GCs proved to consist of three distinct groups, separated by about $120^{\circ}$ and oriented approximately to caudo-rostral, dorso-ventral, and ventro-dorsal (with some caudal component) directions, each group containing DS GCs of ON and OFF subtypes approximately in equal quantity. Thus, this gives six physiological subtypes of DS GCs in total. The six types do not differ from each other in other properties including their RF sizes, shape of the directional tuning curves, and their specificity for stimulus form and speed. All directional tuning curves of DS GCs had cardioid-like appearance, their acceptance angles amounted somewhat more than $180^{\circ}$. All six subtypes of DS GCs have RF sizes of the order of $3^{\circ}-5^{\circ}$. In comparison with other retinal detectors, these units possess finest spatial resolution and thus appeared to be involved in selective aiming to small moving objects in the environment.

Our results indicate that goldfish has six physiologically distinct types of DS GCs projecting to its TO that differ in their selectivity to sign of the stimulus contrast and in preferred directions of movement. The experiments revealed essential differences 
between goldfish DS GCs projecting to the TO and rabbit DS GCs projecting to the superior colliculus. First, ON-OFF type of DS GCs in the rabbit combine ON and OFF pathways, whereas DS GCs in the goldfish use separate ON and OFF channels. Second, the rabbit DS GCs of the collicular projection select four preferred directions separated by about $90^{\circ}$ and aligned with the horizontal and vertical ocular axes, whereas the goldfish ones select three preferred directions separated by about $120^{\circ}$. In this respect, the goldfish DS GCs projecting to the TO resemble the rabbit "slow" DS GCs projecting to the nuclei of the accessory optic system.

At least one of possible targets of DS GCs in the TO is well known. These are DS neurons of the TO itself, which were regularly encountered during experiments. ${ }^{6,8-10,17}$ Their responses are well recognized and distinguishable from those of DS GCs by the pattern of spike train and waveform and tone of individual spikes. ${ }^{9}$ The DS neurons responses were shown to be reversibly eliminated by a topical application of $3 \%$ GABA to the TO surface, whereas DS GCs responses persist. ${ }^{6}$ In contrast to DS GCs, the tectal units are characterized by the enormous size of their $\mathrm{RF}$ and $\mathrm{ON}-\mathrm{OFF}$ character of the responses. The latter feature indicates a convergence of both types (ON and OFF) of DS GCs to such DS tectal neurons.

Coincidence in the number of preferred directions with the number of semicircular canals implies that DS GCs projecting to tectum may be involved in some multimodal sensory integration in postural, locomotor, and oculomotor control in the three-dimensional aquatic world.

\section{ACKNOWLEDGMENTS}

Supported by the Russian Foundation for Basic Research, grant 04-04-49430, and by the Program 2004 of the Department of Biological Sciences RAS "Integrative mechanisms for control of functions in organism."

\section{REFERENCES}

1. Barlow, H.B., R.M. Hill \& W.R LeVICK. 1964. Retinal ganglion cells responding selectively to direction and speed of image motion in the rabbit. J. Physiol. 173: 377-407.

2. JACOBSON, M. \& R.M. Gaze. 1964. Types of visual response from single units in the optic tectum and optic nerve of the goldfish. Q. J. Exp. Physiol. 49: 199-209.

3. CRONLY-Dillon, J.R. 1964. Units sensitive to direction of movement in goldfish tectum. Nature 203: 214-215.

4. VANEY, D.I. et al. 2001. Direction-selective ganglion cells in the retina. In Motion Vision-Computational, Neural, and Ecological Constraints. J. M. Zanker \& J. Zeil, Eds.: 13-56. Springer. Berlin.

5. Oyster, C.W. \& H.B. BARLOW. 1967. Direction-selective units in rabbit retina: distribution of preferred directions. Science 155: 841-842.

6. Zenkin, G.M \& I.N. Pigarev. 1969. Detector properties of the ganglion cells of the pike retina. Biophysics 14: 763-722.

7. Maximova, E.M., O.Yu. Orlov \& A.M. Dimentman. 1971. Investigation of visual system of some marine fishes. Voprosy ichtiologii 11: 893-899 (in Russian).

8. Liege, B. \& G. Galand. 1971. Types of single-unit visual responses in the trout's optic tectum. In Visual Information Processing and Control of Motor Activity. A. Gydikov, Ed.: 63-65. Bulgarian Academy of Science. Sofia. 
9. WARTZOK, D.\& W.B. Marks. 1973. Directionally selective visual units recorded in optic tectum of the goldfish, J. Neurophysiol. 36: 588-604.

10. KAWASAKI, M. \& K. AoKI. 1983. Visual responses recorded from the optic tectum of the Japanese dace, Tribolodon hakonensis, J. Comp. Physiol. A 152: 147-153.

11. MaXimova, E.M. et al. 1975. Physiological mechanisms of the colour constancy. Neurophysiology 7: 21-26 (in Russian).

12. MaXimova, E.M. 1977. Cellular mechanisms of colour constancy. Activ. nerv. sup. (Praha) 19: 199-201.

13. Maximova, E.M. 1999. Colour and spatial properties of detectors of oriented lines in the fish retina. Iugoslav. Physiol. Pharmacol. Acta 34: 351-358.

14. Maximov, V.V., E.M. Maximova \& P.V. Maximov. 2004. Types of direction-selective inputs to the goldfish tectum [abstract], In The $7^{\text {th }}$ Congress of the International Society for Neuroethology, Program and Abstracts: 190. Nyborg, Denmark.

15. GeSTEland, R.C. et al. 1959. Comments on microelectrodes. Proc. IRE 47: 1856-1862.

16. He, S., W.R. LEVICK \& D.I. VANEY. 1998. Distinguishing direction selectivity from orientation selectivity in the rabbit retina. Vis. Neurosci. 15: 439-447.

17. Guthrie, D.M. \& J.R. BAnKs. 1978. The receptive field structure of visual cells from the optic tectum of the freshwater perch (Perca fluviatilis). Brain Res. 141: 211-225. 